\title{
DISCUSSION (Sadakane)
}

SILANT'EV: What are the lowest values of magnetic field one can measure from the Zeeman effect in these hot stars?

SADAKANE: Perhaps the next speaker, Dr. Dworetsky, can answer that, as I do not know the detailed answer.

DWORETSKY: [ barely audible ] About 20 gauss.

ADELMAN: I want to comment on the assumption that the damping constant is about 10 times classical in the optical region, as you have advocated in the ultraviolet. We should abandon that assumption. Better approximations will change the microturbulence $\xi$ by about $0.5 \mathrm{~km} / \mathrm{s}$.

Also, could you tell me where you obtained the information about the instrumental profile for IUE high resolution spectra?

SADAKANE: Data on the instrumental profiles for IUE were privately communicated by $\mathrm{Dr}$. Y. Kondo of NASA.

COWLEY: Would you comment on the reality of the $\mathrm{Fe} / \mathrm{H}$ and metal/H abundance fluctuations in these stars, and in particular, the extent to which we might consider $\alpha$ Lyr (Vega) as a possible borderline case of the $\lambda$ Boo phenomenon?

SADAKANE: I think there is a diversity in metal abundances, especially iron, of about 1.0 dex among superficially normal late $B$ and early $A$ stars. Vega itself may be related to $\lambda$ Boo stars, although it does not satisfy all of the classification criteria for them.

SEVERNY: In one of your tables you seemed to have a difference in chemical composition of $\alpha$ Lyr derived from the visible and UV spectra. What could be the cause of this? Different spectral resolution?

SADAKANE: My results show there are no significant differences in abundances of $\mathrm{Cr}, \mathrm{Mn}, \mathrm{Fe}$, Co and $\mathrm{Ni}$ between the UV and visual regions. Small differences of about 0.3 dex are mainly due to the poor quality of some of the gf values.

POLOSUKHINA: How accurate are the radial velocities?

SADAKANE: We do not really measure true radial velocity from IUE spectra. I reduce my data by measuring the wavelengths of $F e$ II Iines which can be identified, and adjust the observed wavelengths to the laboratory scale. The accuracy depends on the quality, signal-to-noise ratio and $v \sin i$. In the case of $\alpha$ Lyr, experience shows that radial velocities (i.e., wavelengths) can be determined to $\pm 0.05 \AA$ when I use carefully selected lines of $\mathrm{Fe}$ II.

MENDOZA: For the solar abundances you gave in Table 4, what was the wavelength interval used?

SADAKANE: The solar abundances are based on observations in the visual region of the solar spectrum. 


\section{DISCUSSION (Alecian)}

DOLGINOV: In your paper, you use the normal diffusion coefficient in the magnetic field, which is proportional to $1 / \mathrm{H}^{2}$. This form is valid only if you have very quiet conditions, with laminar flows and no currents, etc. However, if there is even a small amount of turbulence or a current, then the diffusion coefficient should be proportional to $1 / \mathrm{H}$ (the so-called Bohm diffusion). Have you considered this possibility?

My second question is similar to the one I asked Mégessier. How is it possible to keep the elements localized for a long time by the field, bearing in mind that an interchange instability exists because the system is like one in which the heavy medium is above the light one?

My third question is, the observations of spots on the stars (by Khokhlova and others) seems to contradict the predictions of the magnetic diffusion separation theory as presented. Can you explain these discrepancies?

ALECIAN: May I begin by answering the third question? At present, computations concerning element diffusion in the presence of a magnetic field at the surface of an Ap star are done with a very simple model, for example assuming dipole or quadrupole fields, but not allowing for the fact that the real magnetic structures are probably more complicated. Also, I do not think that the results on the distribution of patches of elements are precise enough to decide whether or not elements are accumulated in patches or in rings. Elements accumulated in $r$ ings may diffuse along the oblique field lines.

About your second point, I agree that this instability may exist, but we have not computed exactly for what overabundance of Si this may occur. At present, we are only considering simple situations, and in the future more complex physical situations will probably be considered.

MEGESSIER: May I comment on the second question? The situation for Si is not static, but dynamic, and it is not at all like what happens in a glass containing two liquids of different density, one on top of the other. Here, you have a permanent motion, a sort of flow. As far as the observations of patchy distributions of elements is concerned, it should be remembered what Vera Khokhlova said about not being able to determine what is going on more than $45^{\circ}$ from the sub-solar point. It is difficult to say that there are really contradictions between theory and observations.

ALECIAN: I am slightly embarrassed about the first question, because I do not know the answer!

MICHAUD: Could I start with the other questions first? On the second point, we make the calculations in the simplest possible way, and compare with the observations. It is possible that additional turbulence is important. We shall see when we compare with the observations.

The third point was the reason for my question to $\mathrm{Dr}$. Khokhlova on her review paper. I asked if she could exclude rings and she said no. Michaud, Mégessier and Charland (Astron. Astrophys., 103, p. 244, 1981)) showed that existing observations could be fitted as well by 
rings as by spots.

The first point I considered a number of years ago. I now forget the reason we decided to neglect the Bohm formula, as you call it, but it had to do with different physical situations.

KHOKHLOVA: You said that for vertical diffusion the characteristic time is about $10^{4}$ years and for horizontal diffusion it is about $10^{8}$ years. So, when a star starts from the zero-age main sequence, after $10^{4}$ years anomalies appear, but distributed homogeneously. After that $10^{8}$ years are required to form spots by horizontal diffusion. This means that stars in young clusters or associations of age about $10^{6}$ years should not be spotty.

MEGESSIER: It's a bit too schematic. You have to take into account the geometry of the magnetic field. You can't have a regular overabundance everywhere on the surface. You have it near the magnetic equator because where the lines are parallel to the surface Si II is prevented from sinking.

KHOKHLOVA: If the appearance of spots is really connected with the magnetic field, then complex chemical structures force us to think about more complicated magnetic fields than the simple dipole usually assumed.

MICHAUD: The $10^{8}$ years mentioned was needed to get Si back to the poles. The appearance of other spots or rings can be much faster than that, perhaps on the order of $10^{5}$ or $10^{6}$ years. That is the reason for the observational test which was tried for Si.

DWORETSKY: At least one of the three problems you mentioned, the $X-$ rays, apparently should not be a problem after all. I will discuss this in my review paper tomorrow. The X-ray observations of $A$ stars are now interpreted as being due to two causes. For single late B or A stars, the emission follows the scaling law for soft $X$-rays which is obeyed by the earlier $B$ stars. Stronger $X$-ray emission is only seen from A stars with binary companions, and the new interpretation is that it is the later type companions which give the high $X$-ray fluxes. Whether the star is normal or chemically peculiar seems to make no difference; it is only whether it is double that seems to be important. 